\title{
Lattice Imaging of Self-Assembled Monolayers of Partially Fluorinated Disulfides and Thiols on Sputtered Gold by Atomic Force Microscopy
}

\author{
H. Schönherr and G. J . Vancso* \\ University of Twente, Faculty of Chemical Technology, \\ NL-7500 AE Enschede, The Netherlands \\ Received J une 6, $1996^{\otimes}$
}

\begin{abstract}
Thestructure of self-assembled monolayers (SAMs) of various fluorinated disulfides, perfluoroalkylamide thiols, and a mixed alkyl perfluoroalkylamide disulfide on sputtered gold was studied by atomic force microscopy (AFM). AFM, performed both in air and in ethanol, revealed the monolayer structure with mol ecular resolution on the polycrystallinegol d substrates. For all partially fluorinated disulfides containing ester groups, a hexagonal lattice with a lattice constant of 5.8-5.9 $\AA$ was found. A mixed alkyl perfluoroal kylamidedisulfideformed a hexagonal latticeof a slightly larger latticeconstant $(6.1 \AA)$, whereas the lattice observed for fluorinated thiols containing an amide group was either hexagonal $(5.7-5.8 \AA)$ or distorted hexatic $(5.6,6.2,5.6 \AA)$, depending on thelength of the perfluoroal kane segment and theimaging force. The observed deviation from hexagonal symmetry is attributed to the distorting effect of hydrogen bonding between neighboring amide groups within the monolayer. For short perfluoroalkane segments the distortion is observed at low imaging forces, whereas for long perfluoroalkane segments significantly higher imaging forces arenecessary in order to observethedistortion. Theforcedependence of themeasured lattice symmetries for different chain lengths suggests that the AFM tip penetrates into the SAM and probes at least partially the interior of the SAM.
\end{abstract}

\section{Introduction}

Self-assembled monolayers (SAMs) of organic compounds on solid substrates, especially monolayers of thiols and disulfides on gold, havebeen of considerablescientific interest in thelast 2 decades. ${ }^{1-3}$ Thefabrication of defined organicthin films with high local order and tunablesurface functionalities, and thus properties, is very attractive for applications involving organic molecules as functional units (sensors) or involving gold as electrode material (el ectrochemistry). ${ }^{4}$ Furthermore, it is likely that lithographic techniques based on microcontact printing will be used in industry in the near future. ${ }^{5}$

Understanding thestructure of SAM s on the molecular level is very important for particular applications, such as electrochemical sensors, or modified tips for scanning probemicroscopies (SPM). In SPM thesetips can be used either for chemical sensitive scanning force microscopy ${ }^{6}$ or for specific force measurements between a surface and the modified probe. For example, interactions between complementary strands of DNA or theinteraction between proteins have been measured. ${ }^{7}$

In order to understand the basic features of monol ayer structures and the mechanisms of their formation, es-

* Corresponding author.

${ }^{\otimes}$ Abstract published in Advance ACS Abstracts, J une 1, 1997.

(1) (a) UIman, A. Introduction to UItrathin Films, F rom LangmuirBlodgett Films to Self-Assembly; Academic Press: Boston, 1991. (b) Maoz, R.; Sagiv, J . J . Colloid I nterface Sci. 1984, 100, 465. (c) Dubois, L. H.; Nuzzo, R. G. Annu. Rev. Phys. Chem. 1992, 43, 437.

(2) The first SAMs of disulfides on gold were reported by: Nuzzo, R. G.; Allara, D. L. J . Am. Chem. Soc. 1983, 105, 4481.

(3) Recently, functional groups such as amide groups have been incorporated in the alkanechain; see: Tam-Chang, S.-W.; Biebuyck, $\mathrm{H}$. A.; Whitesides, G. M.; J eon, N.; Nuzzo, R. G. Langmuir 1995, 11, 471. (4) (a) Turyan, I.; Mandler, D. Anal. Chem. 1994, 66, 58. (b) Thoden van Velzen, E. U.; Engbersen, J . F.J .; Reinhoudt, D. N. J . Am. Chem. Soc. 1994, 116, 3597. (c) Rojas, M. T.; Kaifer, A. E. J . Am. Chem. Soc 1995, 117, 5883. (d) Zhang, L.; Godinez, L. A.; Tianbao, L.; Gokel, G. W.; Kaifer, A. E. Angew. Chem. 1995, 107, 236.

(5) Wilbur, J . L.; Biebuyck, H. A.; MacDonald, J . C.; Whitesides, G. M. Langmuir 1995, 11, 825 and references therein.

(6) (a) Frisbie, D.; Rozsnyai, L. F.; N oy, A.; Wrighton, M. S.; Lieber, C. M. Science 1994, 265, 2071. (b) Noy, A.; Frisbie, D.; Rozsnyai, L. F.; Wrighton, M. S.; Lieber, C. M. J . Am. Chem. Soc. 1995, 117, 7943. pecially concerning SAM formation as epitaxial crystallization, ${ }^{8}$ well-ordered and well-characterized gold substrates must be used. It is possible to obtain these wellordered and flat surfaces of $\mathrm{Au}(111)$ by special evaporation and annealing techniques ${ }^{9}$ or, al ternatively, by utilizing gold singlecrystals. n-Alkanethiols with very long chains, for instance, exhibit a substrate dependent lattice structure. For SAMs of docosanethiol, this has been demonstrated by low-energy helium diffraction on SAMs assembled onto different gold crystal facets. ${ }^{10}$

Atomic forcemicroscopy (AFM) and scanning tunneling microscopy (STM) studies of monolayers on Au(111) have been reported in the literature. ${ }^{11,12}$ The $(\sqrt{ } 3 \times \sqrt{ } 3) \mathrm{R} 30^{\circ}$ commensurateadlayer structure of $\mathrm{n}$-alkanethiols on Au(111), as proven earlier by electron diffraction ${ }^{13}$ and lowenergy helium diffraction, has been observed by a number of groups. STM has given insight into different superstructures of the $(\sqrt{ } 3 \times \sqrt{ } 3) \mathrm{R} 30^{\circ}$ commensurate adlayer structure of $n$-alkanethiols on $\mathrm{Au}(111)$ which were previously observed by helium diffraction at low temperatures. ${ }^{14}$ STM also established the origin of the etching process (hol eformation in thegol d substrate) which is concomitant with SAM formation. ${ }^{15,16}$

With AFM , theorientation of thelattice of themolecules within the monolayer with respect to the underlying Au-

(7) (a) Florin, E.-L.; Moy, V. T.; Gaub, H. E. Science 1994, 264, 415. (b) Moy, V. T.; Florin, E.-L.; Gaub, H. E. Science1994, 266, 257. (c) Lee, G. W.; Kidwell, D. A.; Colton, R. J . Langmuir 1994, 10, 354.

(8) Any commensurability of theadsorbatelatticewith theunderlying gold lattice implies an epitaxial relation.

(9) U osaki, K.; Shen, Y.; Kondo, T. J . Phys. Chem. 1995, 99, 14117. See also refs 11 and 12 .

(10) Camillone, N., III; Chidsey, C. E. D.; Liu, G.-Y.; Scoles, G. J Chem. Phys. 1993, 98, 4234.

(11) (a) Alves, C. A.; Smith, E. L.; Porter, M. D. J . Am. Chem. Soc 1992, 114, 1222. (b) Pan, J .; Tao, N.; Lindsay, S. M. Langmuir 1993, 9, 1556.

(12) (a) Widrig, C. A.; Alves, C. A.; Porter, M. D. J . Am. Chem. Soc 1991, 113, 2805. (b) Kim, Y.-T.; McCarley, R. L.; Bard, A. J . J. Phys. Chem. 1992, 96, 7416. (c) Delamarche, E.; Michel, B.; Kang, H.; Gerber, Ch. Langmuir 1994, 10, 4103.

(13) Chidsey, C. E. D.; Loiacono, D. N. Langmuir 1990, 6, 682.

(14) (a) Camillone, N., III; Chidsey, C. E. D.; Liu, G.-y.; Putvinski, T. M.; Scoles, G. J . Chem. Phys. 1991, 94, 8493. (b) Camillone, N., III. Chidsey, C. E. D.; Liu, G.-y.; Scoles, G. J . Chem. Phys. 1993, 98, 3503. 
(111) substrate could be determined by the reversible displacement of themolecules when scanning with higher forces $(\approx 300 \mathrm{nN})^{17}$ or by comparing the orientation of the lattice with the orientation of the triangular terraces of thesubstrate. ${ }^{18}$ Incommensuratetail group lattices were reported for SAMs of a variety of thiols and disulfides on $\mathrm{Au}(111)$. Examples include fluorinated thiols (AFM), ${ }^{19,20}$ azobenzene-terminated thiols and disulfides (AFM and STM ), 18,21 disulfides containing ester groups and alkane or fluoroalkane chains $(A F M)^{18}$ or SAMs of discoid triphenylene derivatives (AFM). ${ }^{22}$

In applications, polycrystalline gold, which is usually rich in Au(111) crystal facets, is predominantly used as substrate for SAMs. Defined multilayer structures can begrown ${ }^{23}$ or diacetylene-containing disulfides and thiols can be successfully polymerized in self-assembled monolayers on polycrystalline gol d. ${ }^{24}$ E specially in the latter case a high degree of lateral organization is necessary in order to successfully polymerize these monolayers. In similarly organized systems, like L angmuir-Blodgett (LB) layers, and also in the solid state topochemical photopolymerization of diacetylenes, the necessity of mutual orientation of the diacetyleneunits at a certain angleand a certain distancehas been demonstrated. ${ }^{25,26}$ This means that the molecules in the monolayers must be laterally highly ordered in two-dimensional crystals, even on polycrystalline or sputtered gold.

Butt et al. ${ }^{27}$ investigated thestructureof octadecanethiol monolayers on sputter ed gold by AFM. They observed a lattice which could not be distinguished from the $(\sqrt{ } 3 \times$ $\sqrt{ } 3) \mathrm{R} 30^{\circ}$ commensurate adlayer which is observed on $\mathrm{Au}(111){ }^{18}$ As they were unable to find crystalline areas of gold that are larger than $1 \mathrm{~nm}$ they concluded that the structure of the two-dimensional lattice of the SAM is governed by the chain-chain interaction. ${ }^{28}$

Here we present results of an AFM study of SAMs of a variety of fluorinated disulfides (seestructures in Chart 1) containing ester groups midway in thechain, fluorinated thiols with an amide group, ${ }^{29}$ and a mixed alkyl perfluo-

(15) (a) Poirier, G. E.; Tarlov, M. J . Langmuir 1994, 10, 2853. (b) Delamarche, E.; Michel, B.; Gerber, Ch.; Ansel metti, D.; Güntherodt, H.-J .; Wolf, H.; Ringsdorf, H. Langmuir 1994, 10, 2869.

(16) (a) E dinger, K.; Gölzhäuser, A.; Demota, K.; Wöll, Ch.; Grunze, M. Langmuir 1993, 9, 4. (b) Sun, L.; Crooks, R. M. Langmuir 1993, 9 , 1951. (c) Schönenberger, C.; Sondag-Huethorst, J . A. M.; J orritsma, J .; Fokkink, L. G. Langmuir 1994, 10, 611. (d) McCarley, R. L.; Dunaway,

O. J .; Willicut, R. J. Langmuir 1993, 9, 2775.

(17) Liu, G.-Y.; Salmeron, M. Langmuir 1994, 10, 367.

(18) J aschke, M.; Schönherr, H.; Wolf, H.; Butt, H.-J .; Bamberg, E.; Besocke, M. K.; Ringsdorf, H. J . Phys. Chem. 1996, 100, 2290.

(19) Alves, C. A.; Porter, M. D. Langmuir 1993, 9, 3507.

(20) Liu, G.Y.; Fenter, P.; Chidsey, C. E. D.; Ogletree, D. F.; Eisenberger, P.; Salmeron, M. J . Chem. Phys. 1994, 101, 4301.

(21) Wolf, H.; Ringsdorf, H.; Delamarche, E.; Takami, T.; Kang, H.; Michel, B.; Gerber, Ch.; J aschke, M.; Butt, H.J .; Bamberg, E.J J Phys. Chem. 1995, 99, 7102 .

(22) Schönherr, H.; Kremer, F. J . B.; Kumar, S.; Rego, J . A.; Wolf, H.; Ringsdorf, H.; J aschke, M.; Butt, H.-J .; Bamberg, E.J . Am. Chem. Soc. 1996, 118, 13051.

(23) (a) Evans, S. D.; UIman, A.; Goppert-Berarducci, K. E.; Gerenser, L. J . J . Am. Chem. Soc. 1991, 113, 5866. (b) Freeman, T. L.; Evans, S. D.; Ulman, A. Langmuir 1995, 11, 4411. (c) Evans, S. D.; Flynn, T. M.; Uiman, A. Langmuir 1995, 11, 3811.

(24) (a) Batchelder, D. N.; Evans, S. D.; Freeman, T. L.; Häussling, L.; Ringsdorf, H.; Wolf, H.J . Am. Chem. Soc. 1994, 116, 1050. (b) Chan, K.; Kim, T.; Schoer, J . K.; Crooks, R. M. J . Am. Chem. Soc. 1995, 117, 5875.

(25) Bader, H.; Dorn, K.; Hupfer, B.; Ringsdorf, H. Adv. Polym. Sci. 1985, 64, 1 .

(26) Bloor, D.; Chance, P. R. Polydiacetylenes; Martinus Nijhoff: Dordrecht, 1985; p 1.

(27) Butt, H.-J .; Seifert, K.; Bamberg, E. J . Phys. Chem. 1993, 97, 7316.

(28) $\mathrm{Au}(111)$ can be imaged with atomic resolution in air and under liquid; see: Manne, S.; Butt, H.-J .; Gould, C. A. C.; Hansma, P. K. Appl. Phys. Lett. 1990, 56, 1758.
Chart 1. Structures of the Compounds Investigated in This Study and Their Abbreviations

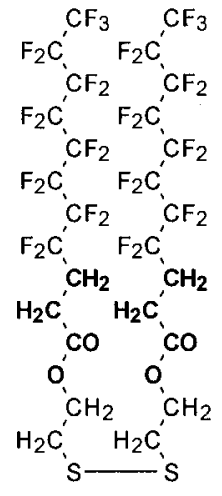

1

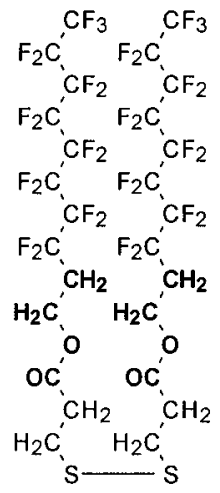

4<smiles>FC(F)CC(F)C(F)CC(F)C(F)C(F)C(F)C(F)C(F)C(F)F</smiles>

7

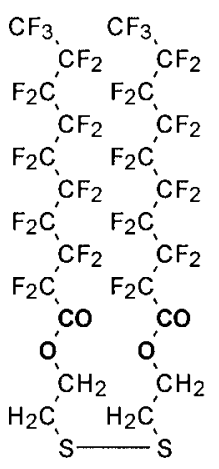

2

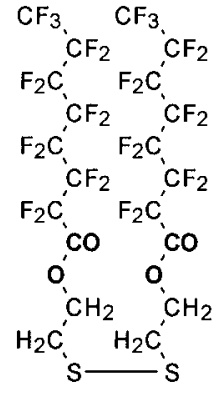

3

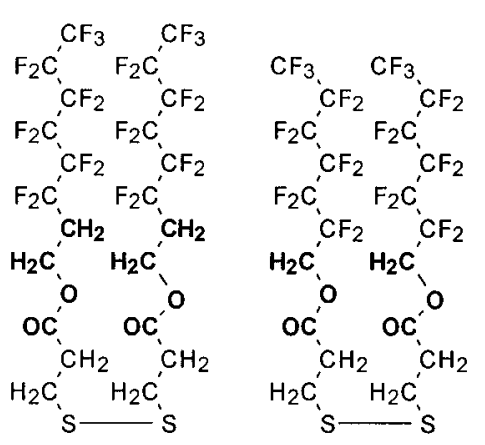

5

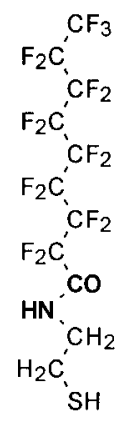

8<smiles>O=C(NCCS)C(F)C(F)C(F)C(F)C(F)C(F)F</smiles>

9<smiles>CCCCCCCCCCCCCC(F)(F)C(F)CC(F)(F)C(F)(F)C(F)(F)C(F)(F)F</smiles>

10 roalkylamide disulfide. ${ }^{30}$ Sputtered gold was used as a substrate. Themain goal was toimagetheSAM structures with molecular resolution on polycrystalline gold and thereby study whether or not local order of SAMs exists on these comparably rough surfaces. In addition, we wanted to compare the observed structure of thefluorinecontaining SAMs with their hydrocarbon anal ogues which werestudied earlier on $\mathrm{Au}(111){ }^{18,31}$ F or thehydrocarbonbased compounds studied on $\mathrm{Au}(111)$, theobserved lattice symmetry was found to depend on the chain length of the alkane segment. ${ }^{31}$

F or SAMs of one of the perfluoroalkylamide thiols (8), results of a computer simulation have been published. ${ }^{32}$

(29) Lenk, T. J ; Hallmark, V. M.; Hoffman, C. L.; Rabolt, J. F. Castner, D. G.; Erdelen, C.; Ringsdorf, H. Langmuir 1994, 10, 4610.

(30) Schönherr, H.; Ringsdorf, H. Langmuir 1996, 12, 3891.

(31) Nelles, G.; Schönherr, H.; J aschke, M.; Wolf, H.; Schaub, M.; Bamberg, E.; Ringsdorf, H.; Butt, H.-J . Submitted for publication. 
Thecompeting interactions within theSAMs, thebinding of the sulfur headgroups to the gold, the intermolecular hydrogen bonding of theamidegroups, and theinteraction of thefluoroal kane chains with each other were shown to result in a uniquestructure. Accordingtothis simulation study, the amide groups formed a meandering structure in themonolayer interior, whilethechain ends werefound to be in a hexagonal array.

\section{Experimental Section}

Synthesis. Compounds 1, 2, 4, 8, and 10 wereavailable from previous studies. ${ }^{18,30}$ Compounds 3, 5, and $\mathbf{6}$ were synthesized in a similar manner to that described in the literature. ${ }^{18,30}$ The chemicals were used as received, except for the fluorinated alcohols which were purified by distillation. Compounds $\mathbf{5}$ and 6 were obtained by reacting 3,3'-dithiobis(propionic acid) with the corresponding partially fluorinated alcohols (Fluorochem), dicyclohexylcarbodiimide (Aldrich), and 4-(dimethylamino)pyridine (Aldrich) in dichloromethane at $0{ }^{\circ} \mathrm{C}$. Compound 3 was synthesized by reacting bis(2,2'-hydroxyethyl) disulfide (Acros) with perfluorooctanoyl chloride (Riedel de Haen) with pyridine as a base at $0{ }^{\circ} \mathrm{C}$. Compounds $\mathbf{7}$ and $\mathbf{9}$ were synthesized in the same way as compound $\mathbf{8}^{29}$ In all cases the final purification of the products was achieved by column chromatography (silica gel, $\mathrm{CHCl}_{3}$ ). The composition of all the products was proven by ${ }^{1} \mathrm{H}-\mathrm{NMR}$ and mass spectrometry (for details, see Supporting Information).

Substrate Preparation. The substrates were obtained by sputtering gold onto freshly deaved mica sheets in a Balzers SCD 040 sputtering machine at an argon pressure of 0.1 mbar. The thickness of the gold layers was estimated to be approximately $100 \mathrm{~nm}$.

Monolayer Preparation. The glassware used was deaned according to procedures described in the literature (seer ef 33 for details and safety regulations). The monolayers were prepared by immersing the freshly prepared substrates into a $5.0 \times 10^{-4}$ $M$ solution of the corresponding compound in dichloromethane (p.a. Merck). After 15-48 h of incubation at room temperature, the samples were rinsed thoroughly with pure solvent and dried in an argon stream. The properties of SAMs of compounds $\mathbf{1}, \mathbf{2}$, 4, 8, and 10 have been characterized in detail before. ${ }^{18,30}$ All SAM s of the compounds studied here exhibited similar contact angles: $\theta_{\mathrm{a}}=115-117^{\circ}, \theta_{\mathrm{r}}=105-107^{\circ}$. The contact angles $\left(\mathrm{H}_{2} \mathrm{O}\right)$ were measured with $\mathrm{Millipore}$ water on a contact angle microscope (Krüss G-1, Hamburg, Germany).

Atomic Force Microscopy. Themeasurements werecarried out with a NanoScope II and a NanoScope III AFM (Digital Instruments, Santa Barbara, CA) in the contact mode. AFM scans were performed both in air, utilizing cantilevers with a nominal spring constant of $0.38 \mathrm{~N} / \mathrm{m}$ (NanoProbe, Digital Instruments), and in ethanol (p.a. Merck), utilizing a liquid cell (Digital Instruments) and cantilevers with nominal spring constant of $0.12 \mathrm{~N} / \mathrm{m}$ (NanoProbe, Digital Instruments). The whole system was allowed to equilibrate for $24 \mathrm{~h}$ prior to measurements in air. For measurements in ethanol, the sample was left in the solvent for $8 \mathrm{~h}$. I maging forces ${ }^{34}$ were minimized to approximately 5-10 nN for measurements in air (except for when mentioned), and to approximately $0.5-1.0 \mathrm{nN}$ for measurements in ethanol. All images shown in this work correspond to raw data obtained in the so-called "height mode" (constant force) which were plane-fitted. Digital filtering was used to eliminate noise (high pass set to 4 , low pass set to 1 ). Thelattice periodicities were observed both with and without the filters.

Weaveraged "up" and "down" scans for quantitative analysis, in order to eliminate the influence of thermal drift. Theimages, which were obtained at different scan rates $(19,26,39 \mathrm{~Hz})$, were analyzed by applying theautocorrelation filter to a selected area

(32) Röthlisberger, U.; Klein, M. L.; Sprik, M.J . Mater. Chem. 1994, $4,793$.

(33) Bain, C. D.; Troughton, E. B.; Tao, Y.-T.; Evall, J .; Whitesides, G. M.; Nuzzo, R. G. J. Am. Chem. Soc. 1989, 111, 321.

(34) (a) Weisenhorn, A. L.; Hansma, P. K.; Albrecht, T. R.; Quate, C. F. Appl. Phys. Lett. 1989, 54, 2651. (b) Weisenhorn, A. L.; Maivald, P.; Butt, H.J .; Hansma, P. K. Phys. Rev. B 1992, 45, 11226. of the resolved lattice. Cross-sectional plots were subsequently laid through theobserved hexatic lattice in threedirections. The repeat length in thesedirections was obtained from thefrequency plots by taking the distance at the maximum spectral density. After multiplication with thecalibration factor, which takes into account theinfluence of sampleheight on measured distances, ${ }^{18,35}$ the observed values were plotted as histograms (see Supporting Information), and the average for each of the three directions was calculated.

\section{Results and Discussion}

The gold substrates, obtained by sputtering, exhibited a granular structure. ${ }^{36}$ The grain size was in the range of $25-50 \mathrm{~nm}$ and varied from batch to batch. Only occasionally was it possible to image a crystalline lattice in the Angstrom scale on top of these gold grains, while it could routinely be observed on Au(111) samples. The lattice imaged on the sputtered gold was identified as $\mathrm{Au}(111)$ based on the analysis of the observed hexagonal lattice (lattice constant of $2.9 \AA$ ). ${ }^{37}$ The size of the crystalline gold patches could not be determined in a quantitative manner. However, we did not observe crystalline order of the gold on length scales exceeding 3 nm by AFM. Compared tothis val ue, the or dered regions of organic mol ecules in SAMs were larger and far more readily and frequently resolved.

Chart 1 shows the structures of the molecules studied in this work. The mol ecular structure of the compounds was varied in order tofind out how thevariations influence thetwo-dimensional lattices of theSAMs. Previous AFM studies on monolayers of compound $\mathbf{1}$ on $\mathrm{Au}(111)$ showed that a hexagonal lattice is formed. ${ }^{18}$ Thus, we are able to compare the results of SAMs on polycrystalline gold with monolayers on $\mathrm{Au}(111)$. Compounds $\mathbf{2}$ and $\mathbf{3}$ have a similar chemical structure but do not possess a hydrocarbon segment. For these molecules, the effect of chain length on the packing into two-dimensional crystals was investigated. A different connectivity of the ester bond and different chain lengths and hydrocarbon segments were incorporated in compounds 4-6. The ester group was replaced by an amide group in thiols 7-9. It has been shown previously that this leads to a stabilization of the monolayer due to intermolecular hydrogen bonding. ${ }^{29}$ In the monolayers of the mixed alkyl fluoroalkyl disulfide 10, the hydrogen bonding is much weaker, due to the net dilution of the amide groups. ${ }^{38}$

We were readily able to image the two-dimensional lattices of all the compounds investigated in this study by AFM. Furthermore, we were able to obtain molecularly resolved images of all compounds on gold even when measuring with higher forces in air. The areas in which an ordered lattice could be imaged were usually smaller than $10-15 \mathrm{~nm}$ across. The averages of the lattice parameters, together with the corresponding standard deviations, are listed in Table 1. First of all the results obtained for the different ester compounds will be discussed followed by a discussion of the results of the fluorinated thiols.

Figure 1 shows an AFM image obtained in air of a SAM of compound $\mathbf{1}$ in molecular resolution. The lattice was determined according to the procedure described in the Experimental Section. The SAMs of compound $\mathbf{1}$ formed a hexagonal lattice with $d=5.8 \AA$. Thevaluecorresponds

(35) Snétivy, D.; Vancso, G. J . Langmuir 1993, 9, 2253.

(36) An AFM image of the granular structure of the sputtered gold surface, as well as an AFM imageshowing the $A u(111)$ latticeis included in the Supporting Information.

(37) The lattice constant of $A u(111)$ is $2.88 \AA .{ }^{28}$

(38) Tsao, M. W.; Rabolt, J . F.; Ringsdorf, H.; Schönherr, H. Unpublished results. 
Table 1. Summary of Observed Lattice Symmetries and Lattice Constants Observed with an I maging Force of $1-10 \mathrm{nN}^{\mathrm{a}}$

\begin{tabular}{lll}
\hline compound & lattice symmetry & lattice constant $(\AA)$ \\
\hline $\mathbf{1}$ & hexagonal & $5.8 \pm 0.2$ \\
$\mathbf{2}$ & hexagonal & $5.9 \pm 0.2$ \\
$\mathbf{3}$ & hexagonal & $5.9 \pm 0.2$ \\
$\mathbf{4}$ & hexagonal & $5.9 \pm 0.2$ \\
$\mathbf{5}$ & hexagonal & $5.9 \pm 0.3$ \\
$\mathbf{6}$ & hexagonal & $5.9 \pm 0.3$ \\
$\mathbf{7}$ & hexagonal $^{\mathrm{c}}$ & $5.8 \pm 0.3$ \\
$\mathbf{8}$ & distorted hexatic $^{\mathrm{b}}$ & $\mathrm{a}=5.6 \pm 0.3$ \\
& & $\mathrm{~b}=6.2 \pm 0.3$ \\
& & $\mathrm{c}=5.6 \pm 0.2$ \\
$\mathbf{9}$ & distorted hexatic ${ }^{\mathrm{b}}$ & $\mathrm{a}=5.5 \pm 0.3$ \\
& & $\mathrm{~b}=6.1 \pm 0.3$ \\
$\mathbf{1 0}$ & & $\mathrm{c}=5.6 \pm 0.3$ \\
$\mathrm{Au}(111)$ & hexagonal & $6.1 \pm 0.3$ \\
& hexagonal & $2.9 \pm 0.1$
\end{tabular}

a Thelattice constants were cal culated as an arithmetic average of all observed values of all measurements and are stated \pm the standard deviation. ${ }^{\mathrm{b}} \mathrm{A}$ limited number of domains shows a hexagonal lattice with $d=5.7 \pm 0.3 \AA$. ${ }^{c}$ By increasing theimaging force to approximately $80 \mathrm{nN}$, the symmetry changes to distorted hexatic: $a=5.6 \pm 0.2 \AA, b=6.3 \pm 0.3 \AA, c=5.5 \pm 0.2 \AA$.

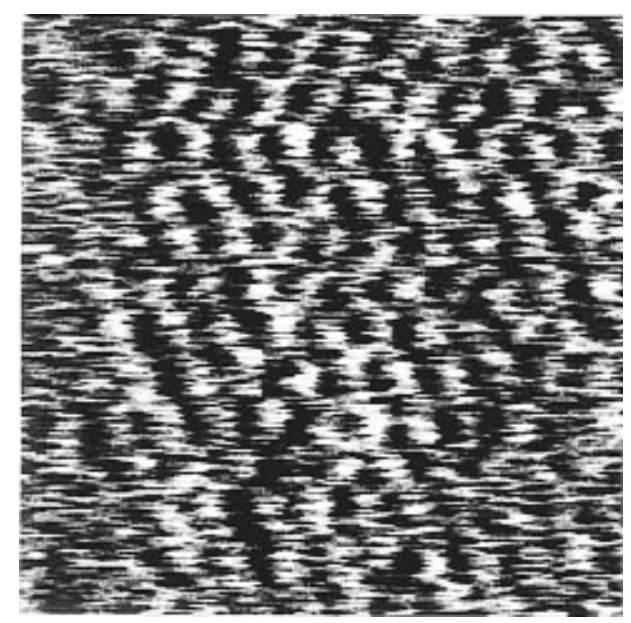

Figure 1. AFM image $(7.0 \mathrm{~nm} \times 7.0 \mathrm{~nm})$ of self-assembled monolayer of compound $\mathbf{1}$ on Au taken in air.

very well tothe value of $5.87 \AA$ obtained on $\mathrm{Au}(111) .{ }^{18} \mathrm{~F}$ or monolayers of $\mathbf{1}$, the standard deviation is the lowest of all the SAMs studied $(\sigma= \pm 0.15 \AA)$. This observation is in agreement with thesmall deviation of theangles of the two-dimensional hexagonal lattice of $\mathbf{1}$ fromtheideal angle of $60^{\circ}$. When $\mathrm{Au}(111)$ is used as a substrate, this deviation of the angle was found to be even smaller than for octadecanethiol. ${ }^{18}$

In Figure 2, an AFM scan of the lattice of a SAM of compound $\mathbf{5}$ is shown. For all the esters, despite their structural differences, we observed a hexagonal lattice with parameters that areidentical to within the accuracy of our data evaluation. We can conclude at this point that the perfluoroal kane segment must be responsible for the formation of the lattice for a number of reasons.

First, wewill consider thelattices found for structurally similar bisalkyl ester disulfides $\left[\mathrm{CH}_{3}\left(\mathrm{CH}_{2}\right)_{n}(\mathrm{CO}) \mathrm{O}\right.$ $\left.\left(\mathrm{CH}_{2}\right)_{2} \mathrm{~S}\right]_{2} \cdot{ }^{18,31} \mathrm{~F}$ or these, theintroduction of theester group midway in the chain is a significant perturbation. Here the packing of the molecules in the lattice is different from n-alkanethiols, for which the repeat unit valueis 5.0 $\AA$. The lattice constant of the hexagonal lattice for compounds with $n=8,10,12$, and 16 is increased to 5.28 $\AA$. For compounds with $\mathrm{n}=10$ and 8, domains with a different latticestructurearealso found. Thesestructures

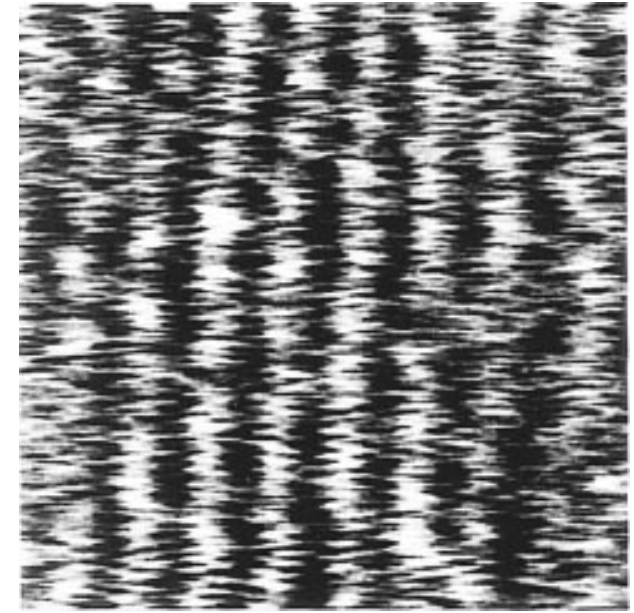

Figure 2. AFM image $(5.0 \mathrm{~nm} \times 5.0 \mathrm{~nm})$ of self-assembled monolayer of compound $\mathbf{5}$ on Au taken in ethanol.

can be described as centered rectangular $(n=10)$ and oblique $(n=8)$. For $n=6$ no crystalline lattice could be imaged. 31

We observed only one lattice for the fluorocarbon analogues. The lattice constant obtained cannot be distinguished from thelattice constant found for partially fluorinated thiols ${ }^{19,20}$ and, as stated above, for compound 1 on $\mathrm{Au}(111)$. Thus, we can conclude that the ester group does not have any influence on the lattice structure for the fluorocarbon-containing compounds studied here. Additional structural changes, like the different connectivity of theester bond, as well as the direct connection of the fluoroalkane segment to the ester group, do not alter the observed lattice. The structure is therefore dominated by the fluoroalkane segment. This can be attributed to thelarge diameter of thefluorocarbon chain and the stiffness of the fluoroalkane segment ${ }^{19}$ (it can be regarded as a "rigid rod"). Thestiffness of thefluoroal kane segment is obvious from a comparison with the abovementioned alkane anal ogues. For an alkane chain with $\mathrm{n}=6$ there was no crystalline lattice observed, whereas for thefluorocarbon chains westill observethehexagonal lattice for segment lengths of $n=5$. For SAM s of partially fluorinated thiols, $\mathrm{HS}\left(\mathrm{CH}_{2}\right)_{2}\left(\mathrm{CF}_{2}\right)_{n} \mathrm{CF}_{3}, \mathrm{n}=5$, on $\mathrm{Au}(111)$, Liu et al. ${ }^{20}$ also observed the lattice by AFM. They described the lattice as incommensurate or at most only close to the high-order commensurate $c(7 \times 7)$ structure.

An AFM scan of a monolayer of the mixed alkyl fluoroalkyl disulfide $\mathbf{1 0}$ is shown in Figure 3. Thelattice symmetry can al so be described in this case as hexagonal, with a lattice constant that was estimated to be equal to $6.1 \AA$. As the lattice constant for dodecanethiol is $5.0 \AA$, and fluorinated thiols have a lattice constant of $5.8 \AA$, an intermediate value was expected. The measured lattice constant value of $\mathbf{1 0}$ seems to be unexpectedly high. Similar high values have been observed for two other mixed alkyl fluoroalkyl disulfides. ${ }^{18}$ Weinterpret this to be a consequence of the unfavorable van der Waals interaction between al kane and fluoroal kanechains. The attractive interaction between neighboring alkane segments, or between neighboring fluoroal kane segments, respectively, is larger than theattraction between alkane and fluoroalkane segments. Such interactions lead to a phase separation in other highly organized systems such as liposomes and monolayers at the air-water interface. ${ }^{39,40}$ In self-assembled monolayers, phase separation cannot be observed for mixed alkyl fluoroalkyl 7687.

(39) Elbert, R.; Folda, T.; Ringsdorf, H.J . Am. Chem. Soc. 1984, 106, 


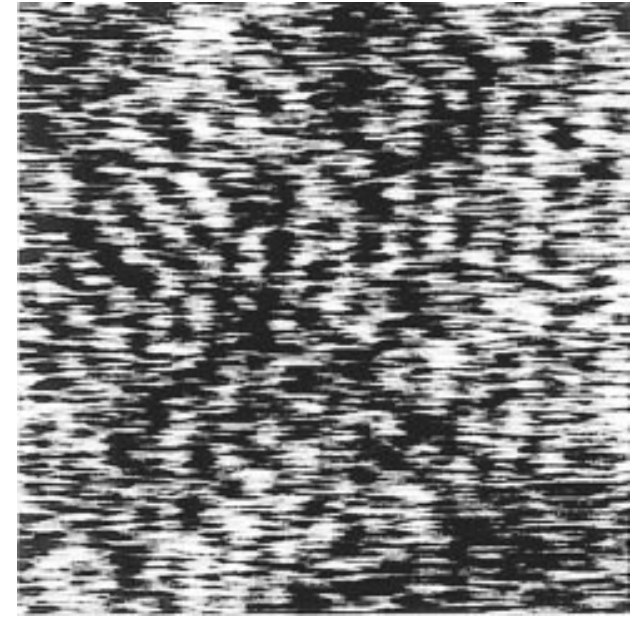

Figure 3. AFM image $(7.2 \mathrm{~nm} \times 7.2 \mathrm{~nm})$ of self-assembled monolayer of compound $\mathbf{1 0}$ on Au taken in air.
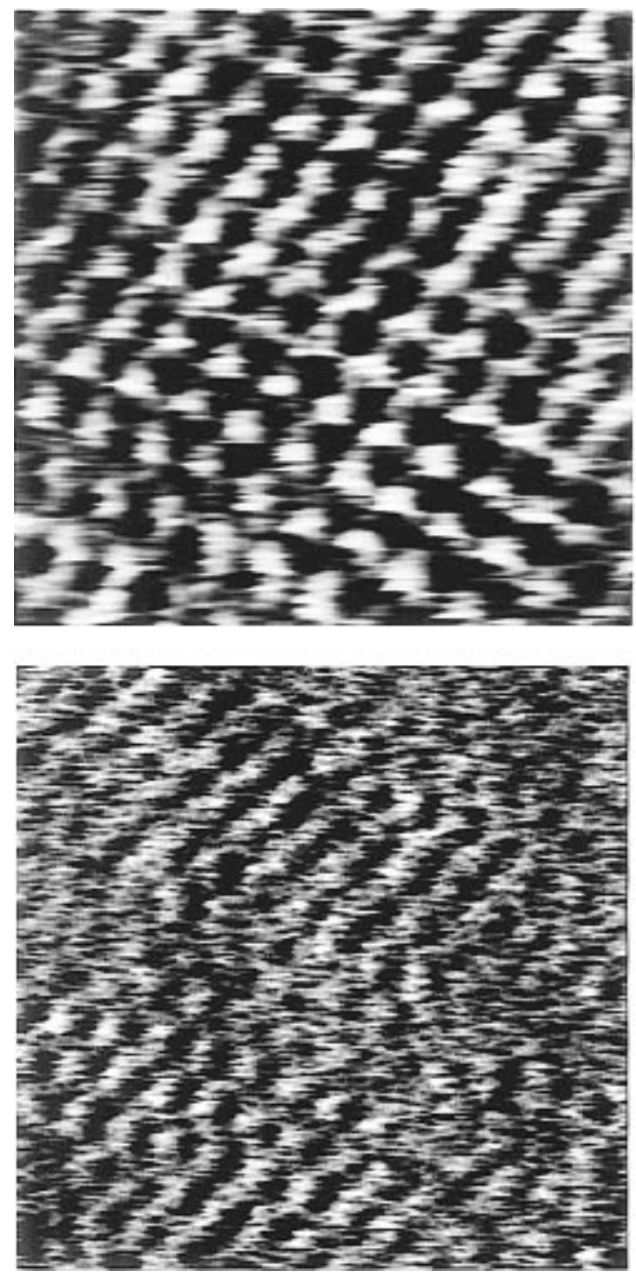

Figure 4. Top: AFM image $(6.0 \mathrm{~nm} \times 6.0 \mathrm{~nm})$ of self-assembled monolayer of compound $\mathbf{8}$ on Au taken in air. Bottom: AFM image $(7.5 \mathrm{~nm} \times 7.5 \mathrm{~nm})$ of self-assembled monolayer of compound $\mathbf{8}$ on Au taken in ethanol.

disulfides-either at room temperatureor after annealing at $100{ }^{\circ} \mathrm{C}$ in air..$^{41}$ The bigger lattice constant of SAMs of compound 10 indicates that both (alkaneand fluoroalkane) chains are incor porated in close proximity in monolayers derived from mixed disulfides. ${ }^{42}$

(40) Overney, R. M.; Meyer, E.; Frommer, J .; Brodbeck, D.; Lüthi, R.; Howald, L.; Güntherodt, H.-J .; Fujihira, M.; Takano, H.; Gotoh, Y. Nature 1992, 359, 133.

(41) Schönherr, H.; Ringsdorf, H.; aschke, M.; Butt, H.-J .; Bamberg, E.; Allinson, H.; Evans, S. D. Langmuir 1996, 12, 3898.
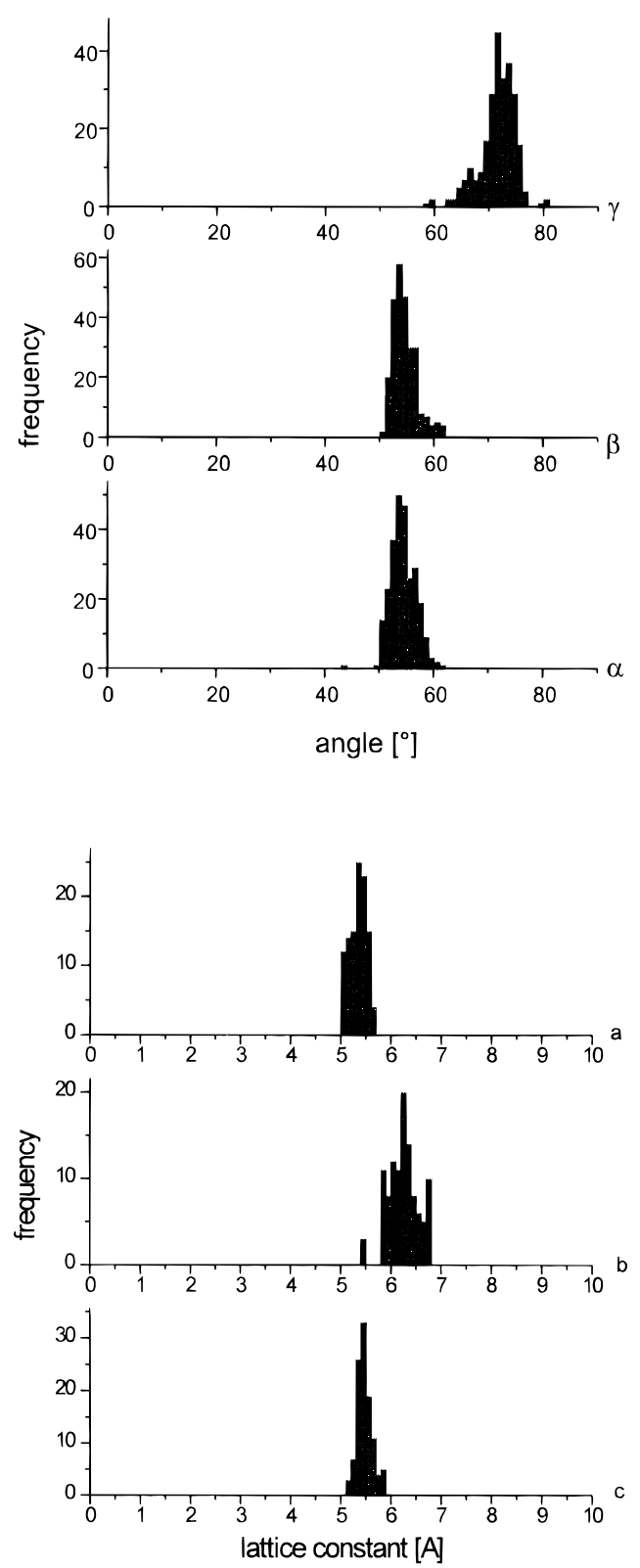

Figure 5. Observed latticeconstants and angles of the distorted hexatic lattice of self-assembled monolayers of thiol $\mathbf{8}$ on Au.

Finally, we examined monolayers of thiols 7-9. Monolayers of compound $\mathbf{8}$ have already been extensively investigated by FTIR, XPS, etc. ${ }^{29}$ A recent molecular dynamics simulation described the structure of SAMs of these molecules. ${ }^{32}$ In addition to the usual factors that influence the monolayer structure, such as the binding of the adsorbate to the surface (bond sulfur-gold) and the chain-chain interaction, these molecules interact via intermolecular hydrogen bonds between neighboring amide groups. The result of the simulation was a closepacked hexagonal lattice of chain ends $\left(-\mathrm{CF}_{3}\right)$ and $\mathrm{a}$ meandering structure of the amide groups.

The AFM image of a SAM of $\mathbf{8}$ shown in Figure 4 (top) was obtained at optimum experimental conditions in air. A nanograph taken in ethanol of a different SAM of $\mathbf{8}$ is shown in Figure 4 (bottom) for comparison. Apart from a few exceptions of hexagonal domains ( $d=5.7 \AA$ ), we usually observed two small and onelargelattice constant

(42) SAMs of mixed methylhydroxyl-terminated disulfides were shown by STM to assemble without phase separation; see: Takami, T.; Delamarche, E.; Michel, B.; Gerber, Ch.; Wolf, H.; Ringsdorf, H. Langmuir 1995, 11, 3876. 
value. For a given domain, the larger periodicity al ways had a certain fixed orientation. I n order to study possible distortions of the hexagonal lattice, we measured the angl es in three directions of the hexatic structure. Both the values obtained for the lattice constant and the measured angles in the three directions of the hexatic latticeareplotted in histograms in Figure 5. Theaverage lattice constant values are very close yet clearly distinguishable, whiletheangles aresignificantly different. The three distributions of the angles do not overlap, and the angl es correspond very well to the values expected on the basis of the measured lattice constants. ${ }^{43}$ Therefore we can conclude, from measurements of both lattice constants and angles, that there is a significant distortion of the lattice. F or SAMs of compound $\mathbf{9}$ we obtained the same results (small number of domains with hexagonal lattice $\mathrm{d}=5.7 \AA$; almost exclusively distorted hexatic lattice, $5.5,6.1,5.6 \AA$ ), whereas SAMs of thiol 7 were shown to have only a hexagonal lattice $(d=5.8 \AA)$.

The structural difference between the molecules 7-9 and the compounds discussed in the previous paragraphs is the amide group. In terms of orientation, the fluoroalkanesegment of themolecules was shown to beoriented nearly perpendicular tothesurface. ${ }^{29}$ Theclosest possible nearest neighbor distance for perpendicularly oriented perfluoroal kane chains is approximately $5.55 \AA .{ }^{44}$ The smallest nearest neighbor distance observed is (within experimental error) consistent with this value. Comparing the surface requirement per $-\mathrm{CF}_{3}$ group with values observed for monolayers at the air-water interface, we can conclude that the observed packing value for SAMs of compound $\mathbf{8}$ on gold is located at the lower end of the range reported by Bernett and Zisman. ${ }^{45}$ These authors measured an area per molecule of $28-32 \AA^{2}$ at the breakdown pressure, whereas in the distorted lattice of compound $\mathbf{8}$ each thiolate has a surface area of $28.7 \AA^{2}$.

On the basis of the findings for the fluoroal kane esters 1-6, we can rule out the possibility that the simple reduction of the symmetry of the molecule from a "rigid rod" to a "rigid rod with fixed kink" is the origin of the lattice distortion. Our hypothesis is that the intermolecular hydrogen bonding is responsiblefor this reduction of the symmetry of the two-dimensional lattice. Since hydrogen bonding is directional, the effect on the packing of the fluoroalkane chains is expected to be anisotropic. The results of the computer simulation described in ref 32 do not seem to agree with our data for compounds 8 and 9 if we postulate that we probe the chain ends with the AFM. However, if we assume that the tip has a considerablepenetration depth intotheSAM, as concluded from previous studies on mixed bisalkyl disulfides with different chain lengths ${ }^{18,46}$ and calix[4]resorcinareneSAMs

(43) Wewould expect $\alpha=56^{\circ}, \beta=56^{\circ}$, and $\gamma=69^{\circ}$ based on geometric considerations; we measured $\alpha=54 \pm 2^{\circ}, \beta=54 \pm 2^{\circ}$, and $\gamma=71 \pm$ $3^{\circ}$.

(44) Polymer Handbook, 3rded.; Brandrup, J ., I mmergut, E. H., Eds.; Wiley: New York, 1989; $\mathrm{p}$ V/37.

(45) Bernett, M. K.; Zisman, W. A. J . Phys. Chem. 1963, 67, 1534

(46) Nelles, G.; Schönherr, H.; Vancso, G. J .; Butt, H.-J . Submitted for publication. on $\mathrm{Au}(111),{ }^{47}$ our observations are in accordance with the simulation results of ref 32 . In this case the AFM tip would partly probe the interior of the SAM, and thus the effect of the hydrogen bonding would be sensed.

In order to support this interpretation, we carried out a series of force dependent measurements with SAMs of 7, which were shown to have a hexagonal lattice when imaged with low forces $(5-15 \mathrm{nN})$. When the forces were increased to about $80 \mathrm{nN}$, a clear lattice distortion was measured. As for the homologous thiols 8 and 9, the orientation of thelarger repeat length remained constant with respect to the scanning direction for a chosen domain. ${ }^{48}$ Thus, by increasing the imaging force, which is concomitant with increased tip penetration depth, the interior of the SAM was probed.

In addition, the observed lattice of the mixed disulfide $\mathbf{1 0}$ is consistent with our hypothesis that hydrogen bonding is responsible for the observed lattice distortion of $\mathbf{8}$ and 9. The mixed disulfide $\mathbf{1 0}$ does not show pronounced hydrogen bonding, ${ }^{38}$ and thus a distorted lattice would not be expected.

\section{Conclusions}

The structure of the two-dimensional lattices of monolayers formed by a variety of partially fluorinated disulfides containing ester groups, a mixed alkyl perfluoroalkylamidedisulfide, and perfluoroalkylamidethiols with different chain lengths on polycrystalline gold was revealed by atomic force microscopy with molecular resolution. Thus, even on sputtered gold, SAM s are formed with a high degree of local order. For all the esters, despite several structural variations, a hexagonal lattice with a latticeconstant of 5.8-5.9 $\AA$ was observed. This indicates that the interaction of the fluoroalkane segments dominates themonolayer structureon polycrystallinegold. The mixed alkyl perfluoroalkylamide disulfide formed a hexagonal latticewith a lattice constant of $6.1 \AA$, whereas the perfluoroalkylamide thiols with a short fluorocarbon segment formed predominantly a distorted hexaticlattice. On the basis of theinsensitivity of thelattices of theesters to structural variations and the observed distortion of the hexagonal lattice of the perfluoroalkylamide thiols with a long fluorocarbon segment after increase of theimaging force, wepostulatethat intermolecular hydrogen bonding is responsible for this distortion.

Acknowledgment. The authors would like to thank Professor Strathmann (University of Twente) for access to the sputtering machine, the University of Twente for financial support, and Mrs. Anne Klemperer for her help with editing the manuscript.

Supporting Information Available: $1 \mathrm{H}-\mathrm{NMR}$ and MS data for all products, AFM images, and histograms (6 pages).

\section{LA960561X}

(47) Schönherr, H.; Vancso, G.J .; Huisman, B.-H.; van Veggel, F . C. J. M.; Reinhoudt, D. N. Langmuir 1997, 13, 1567.

(48) The rotation of the scanning direction resulted in a rotation of the observed lattice, which excludes drift or slippage as the origin of the observed distortion. 